\title{
Thoughts on COVID-19
}

\author{
Colin A. Ross* \\ Institute for Psychological Trauma, 1701 Gateway, Suite 349, Richardson, TX 75080, USA
}

*Corresponding author: Colin A. Ross, Institute for Psychological Trauma, 1701 Gateway, Suite 349, Richardson, TX 75080, USA

Received: July 01, 2020; Accepted: July 03, 2020; Published: July 12, 2020

\section{Introduction}

Despite calls by leading medical authorities and organizations to base policy on science and to trust the experts, statements made by physicians, academic epidemiologists, computer modelers, and the CDC and WHO have been inconsistent and confusing during the coronavirus pandemic. What follows are some thoughts and impressions about the pandemic based on what I have seen in the media. I am commenting only on professionals, and my focus is on the United States.

\section{Death Rates}

Much of the time the death rates stated by individual physicians and health care organizations have been much higher than reality because they are stated as deaths per positive coronavirus test. This has resulted in estimated death rates as high as $13 \%$. These high death rates are an artifact of the number of individuals tested. The death rates should be calculated based on the test-confirmed COVID-19 deaths in the population as a whole. If we assume that $20 \%$ of the population has contracted the coronavirus and there are $330,000,00$ million people in the United States, this would mean that there are $66,000,000$ people who have contracted the coronavirus. If there have been 115,000 COVID-19 deaths, this result in a death rate of $0.17 \%$ per infected person, which is almost the same as the death rate for the flu provided by the CDC. If we assume that only $5 \%$ of people in the United States have contracted the coronavirus, then the death rate is $0.7 \%$ per infected person. This seems to be the realistic range for the death rate for coronavirus infection based on information available to date $-0.1 \%$ to $0.7 \%$. As the number of infected individuals and deaths increases, the death rate may fluctuate a little but should remain in this range. No matter what percentage of the population has been infected to date, the death rate in the entire population so far is $115,000 / 330,000,000=0.03 \%$ or 3 in 10,000 .

\section{Computer Models}

Computer models of the pandemic have been generated by leading epidemiologists at major universities. Early in the pandemic some of these models were predicting 2,000,000 COVID-19 deaths in the United States. In late April, 2020 one model predicted 200,000 deaths in the United States in June alone if lockdown was lifted. If we assume that there will be 200,000 COVID-19 deaths in the United States during the first wave in 2020, then these estimates are 10 times the actual numbers.

\section{The Importance of Testing}

Experts have stated that insufficient testing is a bad thing because we don't know the actual number of infected individuals in the country as a whole or in different regions and cities. This is an epidemiological problem that could be solved with basic sampling procedures requiring a finite and affordable number of tests. We have random sampling of the general population all the time conducted by a variety of organizations. This is a basic failure of medical and public health organizations. It does not instill confidence in medical authorities. Concerns about upward trends in cases post-lockdown rarely take into account the increased amount of testing, and rarely explain to the public that the increase in cases could be at least in part an artifact of increased testing.

\section{Comparison to The Flu}

For both the flu and COVID-19 there are serious and basic problems with how deaths are counted. For both, cases can be counted as virally caused deaths without any testing having been done, or if someone dies from other causes but happens to be coronaviruspositive at the time. This further increases the uncertainty about what is going on, and undermines confidence in authorities and experts.

\section{Facemasks}

In a period of a few months we went from the Surgeon General stating adamantly that facemasks need not be worn by the general public to mandatory facemasks in some states and counties, with no intervening new science. These polar opposite recommendations were backed by federal government health agencies and medical organizations, with no intervening new science. As of June, 2020, the CDC was recommending that asymptomatic people wear masks in public and the WHO was recommending against that.

It is agreed that the coronavirus is about 0.1 micron in size. It is hard to find the pore size of regular surgical masks online but the numbers vary from 50 microns to 500 microns. It is clear that surgical masks will provide no barrier to individual viruses. The same is true for viruses in aerosols. The only possible protection conferred by facemasks is for viruses contained in larger droplets. Why then are medical authorities recommending or even mandating facemasks? The most common rationale is to protect the public from transmission by asymptomatic carriers who might sneeze or cough in public. However, a person with coronavirus in his or her system who is coughing or sneezing is not an asymptomatic carrier. In any case, how often do asymptomatic people 
cough or sneeze in public, with or without a pandemic? It is widely stated by medical authorities that facemasks do not protect an individual from others: the purpose is to protect others from infection by asymptomatic carriers. For instance, the CDC states that, "The cloth face cover is meant to protect other people in case you are infected" (https://www.cdc.gov/ coronavirus/2019-ncov/prevent-getting-sick/prevention.html).

This makes no sense, and is illogical and self-contradictory. If wearing a facemask won't protect me from you, how is it supposed to protect you from me? If persons $A$ and $B$ are both infectious asymptomatic carriers and both are wearing facemasks, the CDC is saying that person A (you) will not be protected but person B will be protected from you. This is a weak motivational message because it hinges on altruism rather than self-protection and it is illogical and unscientific. Mandating facemasks to reduce coronavirus transmission by asymptomatic carriers in public is unlikely to be effective and it could at most have a small effect. For example, in a recent study of coronavirus infection rates in fifteen states and D.C. for 21 days after mandating of public facemasks the infection rate declined by $0.9 \%$ $2.0 \%$ in each five-day increment compared to the five days before the mandate [1]. The time period sampled was March 31 to May 22, 2020, during which time the curve was flattening in most of the country. If we assume that the overall infection rate in the general population was $5 \%$ then the decline was a maximum of $(.05 \times .02=.001) 0.1 \%$ of the population as a whole, and only a portion of that reduction could be attributed to facemasks since many other uncontrolled variables were operating at the same time. There is no stronger evidence concerning the effectiveness of facemasks in the real world. Arguments that masks do no harm do not take into account the burden of hundreds of millions of masks per day on landfills and the environment.

\section{Hydroxychloroquine}

Hydroxychloroquine has oscillated from being a major tool against the coronavirus to being a medication that is contraindicated outside research settings within a few months. The contraindication is due to a combination of lack of efficacy and cardiac side effects and risk of death. Both poles of this oscillation have been endorsed by medical authorities and medical and public health institutions in the absence of adequate controlled trials.

\section{Remdesivir}

Like hydroxychloroquine, remdesivir has been hailed as a major tool against the coronavirus, in the absence of definitive controlled data. Preliminary data indicate that the drug reduces time in hospital from an average of 15 days to 11 days. There are no data showing an effect on mortality or on long-term morbidity. The cost of this drug has been significantly reduced to about $\$ 3000$.00 for a 5 -day course in the US, but it cannot be used at all in populations without access to modern hospitals and IV equipment. The point is not that remdesivir is useless or should be abandoned - the point is that it has been overendorsed by US medical authorities and experts.

\section{Respirators}

A recent study tabulated 5700 COVID-19 patients admitted to hospitals in New York [2]. The paper reported that $97.2 \%$ of
COVID-19 patients over 65 years of age placed on respirators in New York died. The point is not that respirators are useless, but that their importance and effectiveness have been over-endorsed by medical authorities and organizations. The most effective tools, so far, appear to have been basic public health measures like social distancing and self-quarantines. To do a truly scientific analysis of the clinical costbenefit of respirators for COVID-19 patients, we will need follow-up data on morbidity caused by the respirators, which seems to be severe in some cases.

\section{Sweden}

Sweden has been denigrated by US medical authorities for having exposed its citizens to an increased risk of illness and death by not imposing a lockdown. What are the data? If we round off the current numbers, Sweden has a population of 10,000,000 and has had 3,000 COVID-19 deaths. This is a death rate of 0.0003 . The United States has a population of 330,000,000 and has had 115,000 COVID-19 deaths. This is a death rate of 0.0003 . The data actually show that lockdown has no effect on the death rate. As recently as mid-June, Sweden was denigrated for its high number of cases due to insufficient lockdown but its death rate from COVID- 19 was $0.03 \%$ of the population compared to $0.01 \%$ in Germany, $0.02 \%$ in the United States and $0.05 \%$ in Italy. Sweden is the closest thing we have to a control group, imperfect as it is. Yet, US medical authorities will conclude with certainty, in the absence of any rigorous control group, that lockdown saved many lives. In social psychology this is called an attribution error.

\section{Vaccinations and Herd Immunity}

My children and I are all fully vaccinated against childhood infectious diseases. I remember the polio epidemic. The smallpox vaccine has been a major public health success. I am not an antivaxxer to the slightest degree. But our medical authorities are placing too much hope and emphasis on the development of a coronavirus vaccine. It is worth trying, but so far no effective vaccine has been developed against any coronavirus and flu vaccines vary in effectiveness for a given flu season's primary strain from $9 \%$ to $60 \%$ according to the CDC, with an average of $40 \%$ over decades. The odds that we are going to solve the COVID-19 threat through vaccines are low. One of the main arguments in favor of developing a coronavirus vaccine is to generate herd immunity. This has worked for measles, for which there is a herd immunity above $95 \%$ in the United States due to vaccines. According to the CDC, we have tens of thousands of flu deaths in the United States per year even though we have had flu vaccines for years. The flu vaccine provides immunity at a rate far below the threshold for meaningful herd immunity, which is usually said to be about $70 \%$. The evidence to date suggests that a coronavirus vaccine will not confer useful herd immunity. The herd immunity argument in favor of a coronavirus vaccine is not logical or based on science. It is a false hope.

One of the arguments mounted in favor of a coronavirus vaccine by medical authorities and institutions has been that the virus itself may not confer immunity on everyone infected, and if it does the immunity may not last. The argument is that vaccines are required both to protect individuals and to generate herd immunity. This is 
illogical. If the virus does not confer adequate long-lasting immunity, why would we expect the vaccine to do so? We already know that the CDC recommendation is to get a flu vaccine annually because it does not confer long-lasting immunity. The herd immunity argument is another example of statements made by medical authorities that are not based on logic and science. All of these concerns about the effectiveness of a coronavirus vaccine are compounded by recent reports that the virus has already mutated, and the mutated variant is now the primary strain.

\section{Discussion}

The purpose of this commentary is to describe the conflicting, inaccurate and unscientific statements made by medical authorities and medical and public health organizations. These statements cannot be defended by saying that the pandemic situation is fluid - which it is - because the statements are inconsistent with each other, with basic logic and with the available science. Authorities have been contributing to public confusion by making contradictory and unscientific statements about the coronavirus, COVID-19, epidemiology, and the risk-benefit of various actions and inactions while advocating that policy be based on science and data. This cannot help the public or policy makers, and is likely to undermine the public's faith in science and medicine. An over-arching problem is that we still don't know the basic epidemiology of the pandemic, which we could have learned from random testing of the general population. Numbers reported in the media and statements by the CDC and health authorities vary widely in terms of the total number of individuals infected, the number of hospitalizations per infected person, the death rates per infected person and other pieces of basic information. We still lack any controlled studies of the effectiveness of facemasks at reducing infection and death rates in the real world, even though such studies would be easy to conduct. It is very hard to know what is actually going on, given that statements by medical authorities are variable, inconsistent and not based on adequate science. When the CDC and the WHO make opposite statements about the need for asymptomatic individuals to wear facemasks in public, both cannot be right. Although the WHO statement below refers to influenza viruses and the CDC statement to COVID-19, the protective effects of facemasks are the same for both since the viruses are all far smaller than the pore size of masks and cloth coverings worn by the public.

\section{WHO}

"No recommendation can be made at this time for mask use in the community by asymptomatic persons, including those at high risk for complications, to prevent exposure to influenza viruses.

https://www.cdc.gov/flu/professionals/infectioncontrol/ maskguidance.htm

\section{CDC}

"While people who are sick or know that they have COVID-19 should isolate at home, COVID-19 can be spread by people who do not have symptoms and do not know that they are infected. That's why it's important for everyone to practice social distancing (staying at least 6 feet away from other people) and wear cloth face coverings in public settings. Cloth face coverings provide an extra layer to help prevent the respiratory droplets from traveling in the air and onto other people."

\section{References}

1. Lyu W, Wehby GL (2020) Community use of face masks and COVID-19: Evidence from a natural experiment of state mandates in the US. Health Affairs: doi. org/10.1377/hlthaff.2020.00828.

2. Richardson S, Hirsch JS, Narasimhan M, Crawford M, McGinn T, et al. (2020) Presenting characteristics, comorbidities, and outcomes among 5700 patients hospitalized with COVID-19 in the New York City area. JAMA 323: 2052-2059. [crossref]

\section{Citation:}

